\title{
Stated and revealed preferences in companion animal choice
}

\author{
Samantha E. Cohen ${ }^{1} \cdot$ Peter M. Todd ${ }^{1}$
}

Published online: 7 May 2019

(C) The Psychonomic Society, Inc. 2019

\begin{abstract}
Humans often say they prefer certain attributes and trait levels and yet choose options inconsistent with those preferences, a phenomenon known as the stated-revealed preference gap. In this article, we compare preferences and choices in the decision to adopt a dog, a social-choice problem that is largely one-sided. We used existing and newly gathered field data about the dog adoption process to study how people make their choices of companion animals and how those choices can be improved. We found that in the real-world choice of dogs within an animal shelter, individuals generally showed a large amount of overlap between their stated preferences and their ratings of the traits of their chosen dog. However, there was little relationship between an adopter's perceptions of their chosen dog's behavioral traits and third-party in-shelter behavior evaluations of the same dogs, suggesting that it is difficult to predict which dogs will satisfy an adopter's preferences. We also tested which commonly collected factors impact how quickly dogs are adopted from animal shelters. Overall, this work provides insight into the process of combining experimentally collected data and big data to elucidate choice behavior.
\end{abstract}

Keywords Animal shelters $\cdot$ Decision making $\cdot$ Social preferences $\cdot$ Dogs $\cdot$ Human-animal interaction

\section{Introduction}

Humans are social creatures, and the social partners we choose have a strong impact on the quality of our lives. A vast amount of research has studied how people choose each other as romantic and social partners, with much of it intended to help people make better choices with happier outcomes. But fellow humans are not the only social partners that people choose- - a large proportion also select pet animals from whom they obtain companionship and social interaction. In the US, for instance, over a third of households have at least one pet dog, the paradigmatic social animal (American Veterinary Medical Association, 2012). Like our choices of human partners, though, our choices of dogs as companions are not always perfect: Whereas $43 \%$ of marriages in the US will break up within 15 years (Bramlett \& Mosher, 2002), 13\% of dogs adopted from shelters are returned (American

Electronic supplementary material The online version of this article (https://doi.org/10.3758/s13428-019-01253-x) contains supplementary material, which is available to authorized users.

Samantha E. Cohen

cohense@iu.edu

1 Cognitive Science Program and Department of Psychological and Brain Sciences, Indiana University, Bloomington, IN, USA
Humane Association, 2013). These failed choices have consequences: The majority of divorced people do not remarry within 10 years (Bramlett \& Mosher, 2002), and at least $13 \%$ of abandoned dogs in shelters are euthanized (Shelter Animals Count, 2016).

In this article, we use existing (found) and newly gathered field data about the dog adoption process to study how people choose companion animals and how those choices can be improved. We begin by analyzing what traits people visiting an animal shelter say they are looking for in a pet dog, as well as what traits they actually end up with in terms of the dogs they adopt. The potential discrepancy between the two, the stated-revealed preference gap, is one possible challenge to successful dog choice, as has been observed in many other choice domains, including human mate choice. A gap can arise from people not knowing what traits they actually value before they make their choice. But even if people do know (and correctly state) what traits they seek in a dog, their choices can be impeded by an inability to judge those traits accurately - either on the part of the adopter or of third parties (e.g., animal shelter employees) who are trying to assess adoptable dogs in order to help people make better choices. In our second study, we analyze the relationship between adopters' ratings of dog traits and the ratings of those dogs produced by a standard dog behavior assessment tool used in animal shelters. We find little 
correspondence between the two. We discuss the reasons why this might be so and possible ways to improve the process by which adopters choose companion dogs.

\section{Studying social-partner choice}

How should we go about studying the ways that people choose dogs as pets? Given that most dogs are acquired for social companionship (and may even be regarded as closer than family members; Barker \& Barker, 1988), the methods used for studying how people choose human companions are a good place to start in considering canine choice as well. One approach is to ask people what people are looking for in a social partner, yielding their stated preferences, which can be elicited by researchers for study (e.g., Buss, 1989; Li, Bailey, Kenrick, \& Linsenmeier, 2002) or requested when an individual seeks help to find a romantic partner (e.g., via a matchmaking service, blind date, or online dating service). Another method for studying choice is to assess the traits of the actual partners that people choose, yielding their revealed preferences (e.g., for dates-e.g., Kurzban \& Weeden, 2005 - or for marriage partners-e.g., Lykken \& Tellegen, 1993). Settings where people make observable social-partner choices and are also available beforehand to discuss their preferences are ideal for collecting both revealed and stated preferences (respectively), as can be done at speed-dating events among humans (Finkel, Eastwick, \& Matthews, 2007) and at animal shelters for dog adoption, as we study here.

The standard rational expectation is that stated preferences should be in line with revealed preferences. In this case, it would be straightforward to pair up individuals seeking partnership by maximizing how much each partner satisfies the stated preferences of the other. Unfortunately, choice research suggests that such an approach is unlikely to be successful, due to the stated-revealed preference gap, a tendency for individuals to state particular preferences on some criteria but then to make actual choices inconsistent with those statements, revealing different preferences (e.g., Beshears, Choi, Laibson, \& Madrian, 2008). In social-partner choice, the stated-revealed preference gap is seen, for instance, in speed-dating, where individuals report romantic preferences beforehand that do not match the traits of the speed-daters they actually select (Todd, Penke, Fasolo, \& Lenton, 2007).

What might cause stated-revealed preference gaps? Choosers may not know what they truly want, but instead use flawed a priori theories to construct descriptions of their desired choice traits (Nisbett \& Wilson, 1977). Individuals could also change their preferences over time or in response to viewing a choice set. Alternatively, demand effects might lead people to purposely misstate their preferences so they appear more socially appropriate. In terms of external factors, the choice sets that people face (especially in lab settings) might lack enough trait variation for stated preferences to be fulfilled (Li et al., 2013). Competition for options might also limit how well preferences can be matched, especially when population preferences for particular traits are common rather than idiosyncratic. Social-partner choice also has a unique constraint, in that it is typically two-sided, such that both individuals must agree to start a relationship together, which could also reduce the likelihood of stated preferences being met. Such effects have been argued to apply in experimental studies of romantic partner choice (Eastwick \& Finkel, 2008; Li et al., 2013; Todd et al., 2007), though they may have less impact on large real-world mating markets (see Beshears et al., 2008, for a review).

\section{Choosing dogs as social partners}

Just as in romantic partner choice, individuals have preferences for a canine companion. When choosing a dog to adopt, individuals primarily consider appearance and aspects of behavior including personality, behavior with people, and friendliness toward children (e.g., Weiss, Miller, Mohan-Gibbons, \& Vela, 2012). Light-in-color, purebred, small, and young dogs are more likely to be adopted and/or to stay in shelters for shorter periods prior to adoption (DeLeeuw, 2010; Lepper, Kass, \& Hart, 2002; Posage, Bartlett, \& Thomas, 1998). Stray (as opposed to owner-relinquished) animals are also often preferred, due to perceptions that owner-relinquished animals may be more prone to behavior problems (DeLeeuw, 2010; but see Wells \& Hepper, 1992).

Understandably, individuals report that behavior is strongly influential on their adoption choices (Marston, Bennett, \& Coleman, 2005). Adopters often have several ways to observe canine behavior, including within the dogs' kennels or in a oneon-one interaction in a separate area (Weiss et al., 2012). Individuals choosing pets attend to behavioral factors such as prior training, friendliness, and response to household members, including any current pets and children (Siettou, Fraser, \& Fraser, 2014). In terms of predicting adoption success, owners derive greater relationship satisfaction from pet dogs who are higher in openness and agreeableness (Cavanaugh, Leonard, \& Scammon, 2008), but it is unclear whether dogs are chosen on these criteria. Several factors used in dog choice are idiosyncratically valued; for example, the importance of the dog's sex is bimodally distributed, with some adopters valuing it strongly but many viewing it as unimportant (Marston et al., 2005).

Similarity is a positive factor for selecting both human and canine companions. In general, individuals tend to select human social partners with similar appearance and personality (e.g., Thiessen \& Gregg, 1980), though complementarity on some traits can be preferred (e.g., Watson et al., 2004). Owners tend to think their dog resembles them in personality (Turcsán, Range, Virányi, Miklósi, \& Kubinyi, 2012). Several studies have reported that dogs also tend to physically resemble their owners, such that photos of owners and their pets can be 
paired at above-chance levels (Levine, 2005; Nakajima, Yamamoto, \& Yoshimoto, 2009; Roy \& Christenfeld, 2004, 2005). Overall, these aspects of canine partner choice are similar to human partner choice: Physical appearance, personality, and behavior matter strongly in both.

The choice of a pet is, however, distinct from human partner choice in other important ways. In particular, pets are purchased (or adopted) similar to a consumer good, unlike the typical selection process of human social companions. Dog choice is therefore more of a one-sided than a twosided (i.e., mutual) social choice problem, which simplifies some of the choice constraints involved. In two-sided romantic choice, searchers must consider both the quality of a partner and the likelihood of a partner returning romantic interest, based on one's own quality as a potential romantic partner. In this case, the stated-revealed preference gap could be produced by the trade-off of choosing a lower-quality partner who is acceptable and likely to return affection (corresponding to lower fulfillment of one's stated preferences) rather than choosing an ideal partner who might be unlikely to reciprocate one's feelings (corresponding to greater fulfillment of stated preferences). Furthermore, even in many typical one-sided choices (e.g., consumer settings), individuals also must consider a products' price point before purchase (with budget being another chooser-level trait), again leading to an expected difference between stated and revealed preferences.

Dog choice provides a unique real-world context where these trade-offs do not apply. Individuals do not need to be "chosen" by a dog to be able to adopt it, and within animal shelters (and in the present study), prices are generally fixed across available dogs. Overall, adopters do not have to settle for an acceptable dog on the basis of their budget or personal appeal to canines and can freely choose a dog that best fits all their preferences, mitigating one potential cause of the statedrevealed preference gap.

Being able to identify which stated preferences are generally used when choosing dogs would allow for the development of an automatic recommender system that considers how well individual animals meet the stated preferences of visitors. An adopter-dog matching algorithm paired with a rapid survey for assessing adopter preferences (as we develop in this work) would allow visitors to receive custom matches and recommendations for animals to meet, which would save time for both adopters and shelter staff. Such a system could expedite and possibly improve the adoption process, shortening the average length of stay, increasing the number of animals adopted, and decreasing the need for space-saving euthanasia.

\section{Using existing and collected data}

We combine two datasets in order to answer our questions about human choices of dogs as social partners: one collected in the field over a year-long period, and another existing dataset covering multiple years. We begin with a study of the statedrevealed preference gap in individuals intending to adopt a dog (Study 1). We compared the stated preferences of individuals to their ratings of their chosen pet. We then explored how well these subjective perceptions of their animals matched the more objectively obtained trait ratings in a large existing dataset covering all animals within the shelter (Study 2). We also examined the adoption patterns that had been previously recorded over multiple years, to see whether they could be predicted by the objective trait ratings. If individuals use preferences for those objective traits to choose a pet, those dogs with the most preferred trait values should be chosen fastest; that is, they would have the shortest length of stay. Our mixed results demonstrate the challenges of using existing organizational data and trying to connect them with purposefully collected data to provide insight into how to improve the use of both types of data.

\section{Study 1: Stated and revealed preferences}

To explore the stated-revealed preference gap in choosing dogs as social companions, we surveyed animal shelter visitors interested in viewing dogs about their (stated) preferences. We then approached those who decided to adopt (adopters) and asked them to describe their chosen pet (revealed preferences).

\section{Method}

We worked with a municipal animal care and control facility (hereafter, shelter) for a county of around 144,000 people in the Midwestern United States during 2017 and 2018. The shelter placed approximately 2,300 animals in homes in 2016, including dogs, cats, small pets, and stray livestock. At this shelter, animals primarily arrive as strays (i.e., an animal not linked to an owner), owner relinquishments, or transfers from another shelter.

A total of 170 eligible adopters participated; the analysis was limited to the 145 participants who passed attentional checks, completed the entire survey, and provided enough information to locate the records of their chosen dog. Most of the participants were 18-29 years old and identified as women. Additional details can be found in the supplemental materials.

\section{Measures}

Stated preference survey Individuals stated whether or not they had a preference on 13 behavioral and physical traits for dogs (see Table 1). If they had a preference, they indicated the trait level(s) they preferred, out of two or three possibilities. Individuals who chose all levels as preferred were coded as having no preference. This survey was completed by all 
Table 1 Percentages with a stated preference and distribution of preferred trait levels across dog traits for all shelter visitors $(n=1,229)$

\begin{tabular}{lllll}
\hline Trait & Percentage With Any Preference & Trait Levels & & \\
\hline Color & $14 \%$ & Light (white, gray, tan): 26\% & Medium (light brown, red): 46\% & Dark (black, dark brown): 39\% \\
Purebred status & $28 \%$ & Purebred: 21\% & Mixed Breed: $79 \%$ & \\
Sex & $36 \%$ & Female: $56 \%$ & Male: $44 \%$ & Extensive training: $9 \%$ \\
Previous training & $56 \%$ & No training: $8 \%$ & Some training: $91 \%$ & Very: $1 \%$ \\
Nervousness & $58 \%$ & Not at all: $48 \%$ & Somewhat: $57 \%$ & Very: $18 \%$ \\
Protectiveness & $66 \%$ & Not at all: $16 \%$ & Somewhat: $72 \%$ & Very: $53 \%$ \\
Intelligence & $67 \%$ & Not at all: $0.4 \%$ & Somewhat: $57 \%$ & Very: $17 \%$ \\
Easily excitable & $69 \%$ & Not at all: $19 \%$ & Somewhat: $68 \%$ & Senior $(8+): 7 \%$ \\
Age (years) & $73 \%$ & Young (<2): $65 \%$ & Adult (2-7): $45 \%$ & Large: $33 \%$ \\
Size & $73 \%$ & Small: $34 \%$ & Medium: $59 \%$ & High: $8 \%$ \\
Energy level & $74 \%$ & Low: $23 \%$ & Medium: $86 \%$ & Very: $37 \%$ \\
Playfulness & $75 \%$ & Not at all: $1 \%$ & Somewhat: $69 \%$ & Very: $83 \%$ \\
Friendliness & $88 \%$ & Not at all: $0.0 \%$ & Somewhat: $24 \%$ & \\
\hline
\end{tabular}

Percentages of preferred trait levels are amongst those individuals with a preference. Individuals could indicate more than one level of preference on three-level traits, so categories may add to more than $100 \%$.

shelter visitors interested in viewing dogs, including but not limited to adopters $(n=1,229)$.

Revealed preference survey After adopting a dog, participants assessed their chosen pet on 45 behavioral tendencies using a 5-point Likert scale $(1=$ Strongly Disagree, $5=$ Strongly Agree). We focused on eight measures corresponding to the behavioral items in the stated preference survey ${ }^{1}$ :

- Easily excitable: "Is easily excitable"

- Friendliness: "Is sociable"

- Energy level: "Has a lot of energy"

- Playfulness: "Is playful"

- Nervousness: "Is anxious"

- Previous training: "Is well-trained"

- Intelligence: "Is intelligent"

- Protectiveness: "Is protective"

In addition, individuals were asked to indicate the coloring of the dog they chose (light, medium, or dark) as well as the size (small, medium, or large). Shelter staff had assessed the age, breed status, and sex of each dog at the time of intake. The age of one dog was missing. The five Likert levels on the revealed preference survey were mapped to the three categorical stated preference levels as follows: Dogs at the high extreme (5) were coded as Very high levels of a trait; dogs at the

\footnotetext{
${ }^{1}$ When possible, measures were drawn from the extensively validated Dog Personality Questionnaire (Jones, 2008). This includes:

- "Dog is anxious" (Study 1)

- "Dog behaves aggressively towards dogs" (Study 2)

- "Dog ignores commands" (Study 2)

- "Is affectionate" (Study 2)
}

low extreme (1) were coded as Not at all exhibiting that trait; and the remainder (2-4) were coded as displaying that trait Somewhat. Energy level and training each had unique levels. For energy level, the dogs rated at the high extreme were coded as High, those at the low extreme as Low, and the remainder as Medium. For training, the dogs rated at the high extreme were coded as Extensive Training, those at the low extreme as No Training, and the remainder as Some Training.

\section{Results}

The traits with the highest levels of stated preferences (all visitors: Table 1; adopters: Table 2) were friendliness, playfulness, and energy level, whereas the least common were sex, purebred status, and color (see Fig. 1 for adopters, red bars). Among visitors with a preference on a given trait, many traits showed one highly preferred level (energy level, training, playfulness, friendliness, excitability, and protectiveness), meaning that there could be more competition for dogs with that trait level, but a few showed a more even distribution of preferred levels (sex and coloring), indicating idiosyncratic preferences across visitors.

Adopters were less likely than visitors as a whole to have a preference for energy level, excitability, nervousness, and protectiveness. Comparing adopters with a preference to all visitors with a preference, adopters were relatively similar, except for having less of a preference for young dogs, light- and medium-colored dogs, not-at-all nervous dogs, and very protective dogs, and a greater preference for mixed-breed dogs, dark-colored dogs, and somewhat nervous dogs. On average, adopters had preferences for $7.4( \pm 3.5)$ of the 13 traits (ranging from 0 to 13 , with $50 \%$ of the data between five and ten traits). 
Table 2 Percentages with a stated preference and distribution of preferred trait levels across dog traits for the adopter subsample $(n=145)$

\begin{tabular}{lllll}
\hline Trait & Percentage With Any Preference & Trait Levels & & \\
\hline Color & $12 \%$ & Light (white, gray, tan): 18\% & Medium (light brown, red): 35\% & Dark (black, dark brown): 47\% \\
Purebred status & $30 \%$ & Purebred: 5\% & Mixed breed: $95 \%$ & Male: $40 \%$ \\
Sex & $32 \%$ & Female: $60 \%$ & Somewhat: $72 \%$ & Very: $0.0 \%$ \\
Nervousness & $50 \%$ & Not at all: $31 \%$ & Some training: $94 \%$ & Extensive training: 5\% \\
Previous training & $54 \%$ & No training: $8 \%$ & Somewhat: $76 \%$ & Very: $9 \%$ \\
Protectiveness & $61 \%$ & Not at all: $16 \%$ & Somewhat: $66 \%$ & Very: $21 \%$ \\
Easily excitable & $63 \%$ & Not at all: $16 \%$ & Medium: $53 \%$ & Large: $34 \%$ \\
Size & $66 \%$ & Small: $34 \%$ & Medium: $90 \%$ & Very: $46 \%$ \\
Energy level & $66 \%$ & Low: $17 \%$ & Somewhat: $62 \%$ & Senior $(8+): 9 \%$ \\
Intelligence & $69 \%$ & Not at all: $1 \%$ & Adult (2-7): $50 \%$ & Very: $32 \%$ \\
Age (years) & $71 \%$ & Young (<2): $53 \%$ & Somewhat: $73 \%$ & Very: $78 \%$ \\
Playfulness & $76 \%$ & Not at all: $1 \%$ & Somewhat: $28 \%$ & \\
Friendliness & $88 \%$ & Not at all: $0.0 \%$ & & \\
\hline
\end{tabular}

Percentages of preferred trait levels are amongst those individuals with a preference. Individuals could indicate more than one level of preference on three-level traits, so categories may add to more than $100 \%$.

We then calculated for each trait the proportion of all adopters with a stated preference whose preference was fulfilled by their chosen pet's trait level, as indicated by the adopter's own rating of that trait of their dog on the revealed preference survey (Fig. 1, gray bars). We compared these proportions to what would be expected if each individual had been randomly assigned a dog from the sample (using the results of a null model over 10,000 iterations; Fig. 1, blue bars). Although the proportion of individuals choosing a dog that met their stated preferences exceeded chance on all traits, this difference was significant only for sex (Cohen's $d=1.05$ ), size $(d=0.62)$, intelligence $(d=0.36)$, age $(d=0.55)$, and playfulness $(d=0.40)$ ( $\alpha$ adjusted to .003 for multiple comparisons), suggesting that the stated-revealed gap was smaller than would have been produced by random choices, and hence that stated preferences on these traits were used to varying degrees in the decision-making process.

To assess the overall extent of the stated-revealed preference gap among these adopters, we also calculated the distribution of the proportion of preferred (stated) traits that each adopter found (revealed) in their chosen dog (i.e., how many people chose a dog meeting half of their stated trait preferences, how many chose a dog meeting all of their preferences, etc.) and compared this to the distribution of proportions of achieved preferences in a random-assignment null model (see Figs. S1 and S2 in the supplemental materials for the distributions). In these distributions, the stated-revealed preference gap was the mean proportion of unmet trait preferences in the chosen dog (where individuals with no preference on a trait were considered to have had their preference met). Overall, adopters generally had a lower stated-revealed preference gap ( $M=17 \%$ of traits unfilled, approximately two of 13 traits, $S D=13 \%$ ) than would be expected due to chance $(M=25 \%$, approximately three of 13 traits, $S D=$ $0.8 \%$ ) [one-sample $t$ test, $t(144)=7.3, p<.001, d=0.61$ ], indicating that they were picking dogs that were not inconsistent with most of the traits they said they wanted in a dog.

The estimate above reflects the stated-revealed gap but is potentially misleading, because individuals varied in the total number of traits for which they had a preference. Some of our participants had no preferences whatsoever, meaning that their stated-revealed preference gap would be 0 ; this is not an identical comparison to a participant who had preferences on all 13 traits and had them all fulfilled, yielding an identical gap of 0 . To control for variation in the number of traits on which individuals had a preference, we limited calculations of the statedrevealed gap to only the preferences on which an individual had some preference. On average, $29 \%(S D=21 \%)$ of an individuals' stated preferences were unfulfilled by their chosen dog, as compared to $43 \%(S D=1 \%)$ by chance [onesample $t$ test, $t(144)=7.84, p<.001, d=0.67$; see Fig. S2 in the supplemental materials].

\section{Study 2-Effects of subjective and objective traits on adoption}

In the prior study, we found fairly consistent overlap between dog adopters' stated and revealed preferences on important traits. But our ability to accurately assess the stated-revealed preference gap was limited by having to use the adopters' own ratings of their new dog's traits, which they might have adjusted toward their original stated preferences, to maintain appropriate coherence between those preferences and their choices (Riefer, Prior, Blair, Pavey, \& Love 2017). Objective third-party ratings of the traits of all adoptable dogs at the 


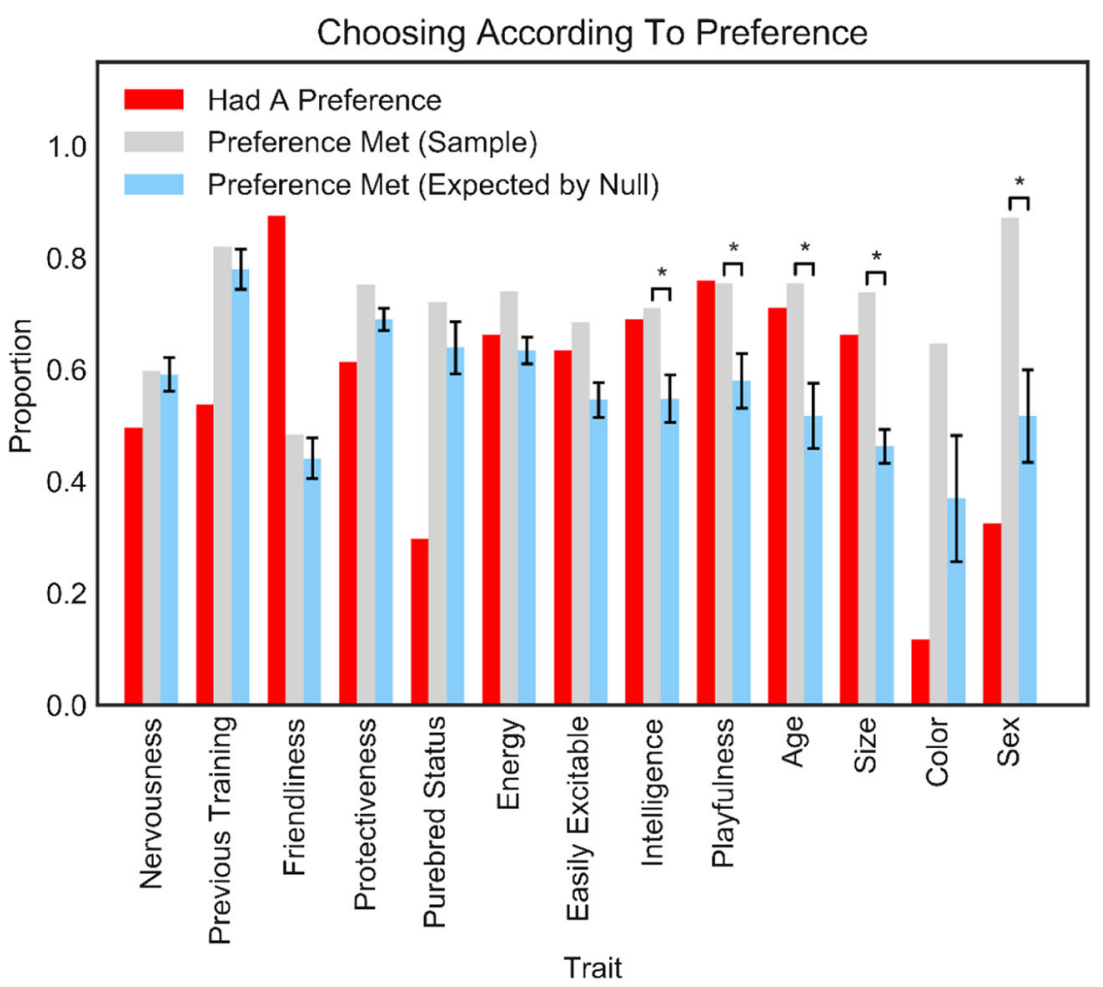

$*: p<0.003$

Fig. 1 Stated and met preferences in dog adoption. The 13 traits that adopters could state a preference for are shown on the $x$-axis. The proportion of adopters expressing a preference for each trait is shown in red. The proportion of those adopters who had a preference on the trait and chose a pet that fulfilled that preference is shown in gray, and the corresponding proportion of such matching choices that would be expected through random choice (using a bootstrapping model of

shelter would enable more accurate comparisons of stated preferences and chosen traits. Moreover, such ratings could be used by shelters to automatically suggest dogs that match a new adopter's preferences, speeding up and improving the adoption process.

Most shelters already use some sort of system to assess the basic characteristics of their animals. But how well these thirdparty ratings of canine behavior traits reflect what adopters are actually looking for must be assessed before those objective traits can be used. In this study, we compared the experimentally collected subjective trait ratings by adopters from Study 1 with a large existing dataset of behavior traits assessed in a more "objective" manner using in-shelter behavior assessments, to see whether the latter can be used to predict dogs that would meet the former set of adopters' revealed preferences. We also examined how the objective traits related to the dogs' length of stay at the shelter before adoption, which is a proxy for the overall attractiveness of the dog within a choice set. Dogs that best fit more adopters' underlying preferences should be desired by more individuals, leading to shorter lengths of stay. The traits most predictive of length of stay
10,000 iterations) is in blue. The $x$-axis is ordered according to the size of the difference between the gray and blue bars - that is, the difference between the proportion of adopters achieving a preference they held in their dog and the proportion who would achieve it due to chance. For all traits, the sample chose dogs that fulfilled their preferred levels on a trait more often than would be expected due to chance alone, though this result was significant only for five traits

constitute revealed preferences at a population level, which can be compared with the stated preferences also at a population level, as we captured in Study 1. Furthermore, if we have both subjectively stated preferences and objectively revealed preferences for particular dogs, we can assess the statedrevealed preference gap at the individual level in a way that gets around the problem of adopters rating their own dogs that we encountered in Study 1.

\section{Method}

\section{Data}

The objective dog traits at our field site (collected January 2016 to March 2018) come from Match-Up II, the shelter data management software with proprietary behavioral testing maintained by the Animal Rescue League of Boston (http:// matchupii.arlboston.org). Match-Up II includes physical trait information and measures of behaviors exhibited in the prior home, within the shelter, and on an in-shelter behavior evaluation. The latter is a battery of 16 subtests that was specifically 
designed to follow scientifically rigorous standards for testing behavioral differences (see the supplemental materials for discussion of the tests and descriptive statistics). The assessments are generally intended to define dog behavior (i.e., responses to specific stimuli; Mirko, Doka, \& Miklosi, 2013), rather than to assess personality (the dog's overall response to the environment), though Match-Up II does attempt to calculate six measures of personality (see below).

\section{Procedure}

On each Match-Up II subtest administered to the dogs in our dataset, shelter staff recorded the presence or absence of each of up to 63 relevant behaviors, movements, or postures, 16 of which were not exhibited by any dog in these data. The occurrences of the 47 behaviors seen in these dogs are shown in Table S1 in the supplemental materials. We excluded the subtests dealing with food possessiveness, canine interaction, and leash manners, since adopters were unlikely to view their new dog in those situations before giving their own subjective trait ratings (see also Mohan-Gibbons et al., 2018, on the limited benefits of food-guarding tests). Some subtests may not have been completed in full (e.g., if the dog snaps and growls during the Strange-Looking Individual subtest, the test might be discontinued for handler safety). If a dog had multiple records within Match-Up II, only the last was kept. Dogs with a stated outcome of "other" or those transferred to another organization were excluded. Occasionally, demographic variables (e.g., coloring) were missing or coded as "Don’t Know."

Predefined personality measures Match-Up II algorithmically calculates friendliness (with a possible range of 0 to 34 ), fearfulness ( 0 to 36 ), excitability ( 0 to 33 ), playfulness ( 0 to 21 ), trainability ( 0 to 15$)$, and aggressiveness ( 0 to 36 ). We excluded aggressiveness due to known methodological issues in "diagnosing" aggressive behavior (Patronek \& Bradley, 2016), and replaced trainability with a less subjective measure, total commands followed.

Total commands followed We counted the total number of commands completed on the Cues subtest (up to five). The five cues on the test were come, sit, sit/stay, paw/shake, and down. Each dog was given up to three chances to complete a cue successfully. Dogs completing zero cues were rated as having No Previous Training; those completing one to three cues were rated as Some Training; and dogs completing four or five cues were rated as Extensive Training.

Coloration Coloration was obtained using separate techniques for the sample of dogs chosen by our adopters in Study 1 and the remaining dogs adopted in our analysis of length of stay (see below). For the dogs from our Study 1 sample, coloration was obtained using existing photographs of those animals $(n=$
120 of 136 unique animals) taken by shelter staff during the animals' shelter stay. Each photo was evaluated by one of two research assistants, who were provided with the three color groups and examples described in Study 1 and asked to provide a primary (and if another predominant coloring belonged to a separate color group, a secondary) coloration group. If no photos were available, coloration was based on primary and secondary (if any) coloration stored within the shelter management database, Animal Shelter Manager.

\section{Measures for predicting length of stays}

We also examined an extended sample of all dogs adopted during the Study 2 period (January 2016 to March 2018), to determine what traits predicted length of stay. A total of 1,746 dogs were adopted during the study period, of which 1,461 had at least one complete and clear length of stay resulting in adoption (see below). Of these 1,461 dogs, 904 had Match-Up II records including behavior evaluations. Procedures varied slightly for the extended sample, as compared to the adopted dogs from Study 1. The limited number of dogs who entered the shelter through confiscation or had an unspecified "other" method of intake were excluded.

Coloration Lacking photos, we used the primary and secondary (if any) coloration provided within Match-Up II. The colors were simplified to the three groups used by adopters in Study 1: dark (black, chocolate, brindle, or liver), medium (brown, gray, red, tricolor, or blue/red merle), and light (cream, white, tan, gold, fawn, buff, gray, or apricot). One animal had a secondary coloration of "other," and this record was excluded from the analysis of secondary coloration.

Size In Match-Up II, size is not directly measured, and weight was rarely included in records by staff, so instead we approximated size using the dog's closest resembled breed and American Kennel Club estimates of the typical weight of a purebred male of that breed: A dog up to 15 pounds $(6.8 \mathrm{~kg})$ was classified as small, one from 15 pounds $(6.8 \mathrm{~kg})$ up to 49 pounds $(22.2 \mathrm{~kg})$ was medium, and one over 49 pounds $(22.2$ $\mathrm{kg}$ ) was large.

Length of stay The total length of stay at the shelter was calculated as the length in days from intake to adoption. Days spent in foster care were then subtracted from the total length of stay; however, the dogs in the system prior to April 2016 utilized a different foster care system that obscured the date that the foster animal returned to the shelter, so dogs in foster care prior to this date were excluded $(n=152)$, as were 47 dogs whose entrance and exit dates were chronologically unclear, and 86 with no completely observed period from intake to adoption (i.e., a dog entered the shelter prior to the study period, but exited during it). Dogs who entered as strays 
had their relinquishment hold (during which an owner could reclaim a lost pet, five days) also subtracted from their total length of stay. Two outliers with a length of stay beyond 150 days were excluded, leaving 1,461 dogs, with 904 completing Match-Up II behavior evaluations. Those animals with multiple visits to the shelter (i.e., returns; $n=136$ ) were included as two or more separate observations and had each visit paired with the same behavioral record, producing an additional 156 records, for a total of 1,060 records.

\section{Results}

\section{Comparison between subjective and objective trait ratings}

Of our sample of 145 adopted dogs in Study 1, a total of 103 dogs had a Match-Up II behavioral evaluation completed, and we limited our analysis to this subset. We ran Spearman's correlations between the subjective (revealed) personality trait ratings of dogs by their adopters and the objective measures of those traits in Match-Up II for the traits friendliness, fearfulness, excitability, and playfulness, shown in the top four rows of Table 3. There was a significant positive correlation between objective and subjective excitability, and a sizable (but not significant) negative correlation for fearfulness. All other correlations were not significant at an adjusted $\alpha$ of .005 for multiple comparisons.

To test whether this surprising lack of correspondence between the subjective and objective measures of these four traits was due to imprecision in the definitions of adjectives such as "friendly" or "fearful," we chose five additional items on the revealed preference survey with fairly straightforward interpretations to simple behaviors measured in Match-Up II (see the bottom five traits in Table 3). We again calculated Spearman's correlations between each revealed preference rating by adopters and the corresponding Match-Up II behavioral criteria. No correlation was significant at the .005 level.
Together, these results suggest that the correspondence between how adopters and Match-Up II evaluate a dog's behavior is far from clear.

Finally, we tested whether there is a greater correspondence between what adopters and Match-Up II raters say about presumably clear and constant appearance traits, in contrast to the potentially variable behavioral traits above. Comparing the primary color of a dog's coat indicated by their adopters $(n=$ 139 ) with the primary (and if available, secondary) color from shelter records, $16 \%$ of adopters categorized their pet's primary coloring differently from the Match-Up II raters (i.e., not matching either the primary or secondary coloring), again indicating a surprising level of mismatch between adopters' and third-party raters' assessments of dog traits.

\section{Predicting length of stay}

Although the Match-Up II ratings did not match directly with individual adopters' own revealed preferences, this could have been due to adopters not accurately assessing their chosen dog's traits or clearly revealing their preferences for those traits. Thus, the more objective ratings given by the trained raters using Match-Up II could still be a useful way to get at traits that are important for adopters making their dog choices. To find out whether the large existing dataset of Match-Up II ratings could be used to identify traits that matter to adopters at a population level, we tested the strengths of various traits from Match-Up II to predict the length of stay of dogs at the shelter until adoption.

Here, inverse length of stay was taken as a proxy for the desirability of (and, hence, the revealed preference for) a dog. We ran an ordinary least squares regression model in Python 3 using the Statsmodels module (Seabold \& Perktold, 2010), dropping any canine stays that did not have all traits defined $(n=41)$, yielding a total of 1,019 stays. We included as numeric predictors age, total commands followed, friendliness,

Table 3 Correlation between trait levels as rated by adopters and assessed by Match-Up II behavior evaluation

\begin{tabular}{llcc}
\hline $\begin{array}{l}\text { Adopter Perceived Trait } \\
\text { (Revealed Preference Survey) }\end{array}$ & $\begin{array}{l}\text { Match-Up II } \\
\text { Calculated Trait }\end{array}$ & Spearman's $r$ & $p$ \\
\hline Is anxious & Fearfulness & -.23 & .02 \\
Is easily excitable & Excitability & .32 & 103 \\
Is playful & Playfulness & -.002 & .0009 \\
Is sociable & Friendliness & .98 & 103 \\
Is well-trained & Total commands followed & .15 & .14 \\
Barks frequently & Number of subtests in which "bark" was exhibited & .60 \\
Behaves aggressively & Total behaviors present on the Dog-to-Dog subtest from the growl, piloerection, & .03 & .03 \\
$\quad$ towards other dogs & inhibited bite, uninhibited bite, show teeth, snap, and head whip categories & .43 \\
Ignores commands & Total commands followed & 103 \\
Is affectionate & Number of subtests in which "lick" was exhibited & .73 & .07 \\
\hline
\end{tabular}

One value significant at $\alpha=.005$ is in bold. 
fearfulness, excitability, and playfulness (see Table 4). We also included the following binary predictors (as dummy variables): coloring (dark and medium relative to light), sex (male), purebred, stray, and size (large and small, relative to medium).

The model was a poor fit overall, with an adjusted $R^{2}=.08$, $F(13,1005)=6.2, p<.001$. Friendliness weakly reduced the length of stay, while surprisingly, playfulness increased the length of stay. Stray dogs, which are often preferred to owner-relinquished dogs due to a lower perceived rate of behavior issues, also exhibited shorter lengths of stay. Finally, large dogs had significantly longer stays, and smaller dogs had shorter stays, relative to medium dogs. Coat color did not significantly impact length of stay; we failed to replicate the black dog effect, in which dogs with dark coloring have longer lengths of stay (note that not all preferences were expected to linearly affect length of stay, because as we found in Study 1, some traits showed a greatest preference for the middle level, including age, size, energy level, previous training, excitability, and protectiveness). On the basis of these results, it is difficult to argue for the usefulness of Match-Up II ratings to predict much about dog desirability at the population level.

\section{Discussion}

\section{Summary of results}

We examined how the stated preferences of individuals adopting a dog from a shelter compared with their revealed preferences as expressed through their own ratings of their chosen dog. On every trait, individuals with a preference were more likely to fulfill that preference than would be expected through chance alone, indicating that they were able to match their stated preferences to their choices reasonably well (especially for age, sex, size, playfulness, and intelligence). Surprisingly, the most valued traits were not necessarily fulfilled at above-chance levels (e.g., friendliness).

However, using individuals' own ratings of their chosen dog is not an objective way of establishing their revealed preferences, so we next examined how well adopters' perceptions of personality and behavior matched those made using a common objective behavior evaluation tool, Match-Up II. There was little clear relationship between the two, which makes it difficult to use the objective system for recommending adoptable dogs to potential adopters on the basis of trying to match their preferences. Furthermore, we tested whether any of the traits measured by Match-Up II can predict the choices of adopters at the population level in terms of length of stay, a measure of canine desirability. Stray and small dogs had shorter lengths of stay, but most behavioral traits did not have much effect. Interestingly, increased playfulness, a desirable trait that was fulfilled above chance for our adopters, increased length of stay on average. Adopters generally indicated they wanted a somewhat playful dog, rather than a very playful dog - it may be that high Match-Up scores on playfulness indicated very high levels of play, which could be indicative of both young age and high energy level (which could be unattractive to many adopters). The unclear relationship between stated preferences for certain trait levels and

Table 4 Results of ordinary least-squares regression predicting length of stay

\begin{tabular}{|c|c|c|c|c|c|}
\hline Variable & $\beta$ Coefficient & $S E$ & $t$ & $p>|t|$ & $95.0 \% \mathrm{CI}$ \\
\hline Constant & 14.862 & 1.623 & 9.159 & $>.001$ & 11.678 to 18.046 \\
\hline Friendliness & -0.1659 & 0.072 & -2.306 & .021 & -0.307 to -0.025 \\
\hline Playfulness & 1.6813 & 0.331 & 5.077 & $>.001$ & 1.031 to 2.331 \\
\hline Age & 0.2553 & 0.135 & 1.886 & .06 & -0.01 to 0.521 \\
\hline Large (dummy) & 3.5507 & 0.897 & 3.958 & $>.001$ & 1.79 to 5.311 \\
\hline Small (dummy) & -2.5819 & 1.291 & -2.0 & .046 & -5.116 to -0.048 \\
\hline Excitability & -0.101 & 0.167 & -0.605 & .546 & -0.429 to 0.227 \\
\hline Total Commands Followed & 0.0075 & 0.24 & 0.031 & .975 & -0.464 to 0.479 \\
\hline Fearfulness & 0.1945 & 0.139 & 1.395 & .163 & -0.079 to 0.468 \\
\hline Dark Coloring (dummy) & -0.6674 & 0.861 & -0.775 & .439 & -2.357 to 1.022 \\
\hline Medium Coloring (dummy) & -0.1969 & 0.983 & -0.2 & .841 & -2.125 to 1.731 \\
\hline Male (dummy) & 1.0554 & 0.751 & 1.405 & .16 & -0.419 to 2.53 \\
\hline Purebred (dummy) & -0.0154 & 0.832 & -0.019 & .985 & -1.648 to 1.617 \\
\hline Stray (dummy) & -3.0355 & 0.938 & -3.235 & .001 & -4.877 to -1.194 \\
\hline
\end{tabular}

Values significant at $\alpha=.003$ (for multiple comparisons) are in bold. Variables are ordered according to their importance to visitors, as measured by the proportion of visitors with a preference on the variable (excluding stray, which was available in the dataset but not included on the stated preference survey as a preference dimension for visitors). 
outcomes within the shelter calls into question just what those shelter tests are measuring and whether they are useful for guiding adoption decisions.

\section{Implications for animal shelters}

A major motivation for this work was to examine which preferences individuals tend to fulfill when adopting a dog, so that shelters may focus their efforts on matching potential pets to visitors. Surprisingly, despite an expectation of a statedrevealed preference gap, most individuals with a preference tended to think their dog fulfilled their preferences, and on average, only about $30 \%$ of trait preferences were unmet — but even choosing randomly, a given dog fulfilled an average of $57 \%$ of any individual adopters' trait preferences. Using the standard definition of a stated-revealed preference gap including traits on which individuals had no preference, the stated-revealed preference gap in our sample dropped to about two of 13 traits, where a random dog fulfilled about three.

If individuals do use their preferences to choose dogs, ideally, those preferences could be used to suggest dogs that will likely satisfy adopters. Data that are already collected could be leveraged to make these suggestions if later adopters are likely to draw the same conclusions about a dog. Indeed, a stated goal for Match-Up II, according to the training manual, is "to help shelters learn about the personality and needs of each dog so that behavioral interventions can be implemented and successful matches can be made" (Animal Rescue League of Boston ${ }^{\odot}$ 2016, p. 3). It appears that the last part of this goal, at least, is not being met in the shelter situation we studied. There was little relationship between the ratings of a dog's friendliness, trainability, or playfulness by the dog's adopter and the corresponding Match-Up II ratings. Practically, attempting to match individuals with dogs that meet their preferences according to Match-Up II's calculations is unlikely to result in the feeling that one's preferences have been satisfied. Why might a disconnect occur between the trait assessments of adopters and Match-Up II?

Nonexperts often confuse different types of dog behavior (e.g., friendliness, playfulness, and submission), report behaviors that are not present (such as growling or nuzzling), or mistake basic characteristics of animals such as age (Tami \& Gallagher, 2009). Our adopters also showed differences from shelter records for even basic qualities of their chosen pets such as coat color. Similarly, when nonexperts evaluate dogs, they attend to a limited number of cues and associate them with a single emotional state, although experts use these cues in tandem with others to differentiate between multiple different dispositions-for example, tail wagging is commonly thought of by nonexperts as a clear indicator of friendliness, when empirically, it is associated with aggression, confidence, defensiveness, and friendliness (Tami \& Gallagher, 2009). Interestingly, in Match-Up II (a purported scientifically driven behavior evaluation), tail wagging is limited to predicting friendliness, matching the nonexpert conception, but in the process missing out on the other traits the behavior could be signifying. The simplification of this cue in the behavior evaluation suggests that practical limitations of implementing a test in a real-world environment might impede the quality or accuracy of behavioral results.

Perhaps some forms of behavior evaluations are accurate in their assessment of dogs' behavior in the shelter environment but are limited in their scope of usefulness beyond the shelter. Patronek and Bradley (2016) eloquently noted that, despite the widespread use of behavior evaluations, it is unclear why one would expect the dog's performance in the evaluation situations to translate into regular interactions with adopters.

\section{Overcoming the stated-revealed preference gap}

Nonexperimental existing data generally consist of revealed preferences (e.g., purchasing behavior), which are often assumed to be the ideal way to assess true preferences. But such revealed preferences generally consist of the choices made under the constraints of the real world, with time limitations, competition, and options that might not span the spectrum of desired traits (Beshears et al., 2008). Stated preferences, in contrast, are made under more controlled conditions but can show other sorts of bias, especially recall. In this work, we utilized both types of preferences to elucidate what matters in choosing dogs. As in mate choice, we found similar tendencies for some traits to be relatively universally preferred, and so subject to competition in revealed preference choice situations (in dogs: friendliness; in humans: kindness and attractiveness-Buss, 1989) and some that are idiosyncratic (in dogs: sex; in humans: religiosity—Shackelford, Schmitt, \& Buss, 2005).

However, dog choice differs from romantic partner choice in several ways that might elucidate the cause of the statedrevealed preference gap. Humans often have extensive experience choosing and observing potential mates, starting in adolescence (e.g., Furman, 2002; Penke, Todd, Lenton, \& Fasolo, 2007). It is unclear whether most individuals have the same plethora of experience in dog ownership, meaning that they might have a poor idea of how particular traits would impact their pet satisfaction and so could make revealed choices inconsistent with their stated preferences. Furthermore, at the time of choice, adopters have only experienced a dog's behavior for a short period in a novel setting, which might not provide an accurate representation of how that behavior would manifest in day-to-day life or how it might change over time (and hence might not give enough information for adopters to tell whether this dog meets their stated preferences). For example, short exposure to the typical puppy behavior of chewing on hands might be tolerable or endearing, but on a daily basis could lose its charm and develop into a life-long habit. 
Overall, as compared to other social choices, individuals might lack the same quantity of experience in the dog domain, and with it useful feedback to revise their stated preferences, both of which could produce unsatisfactory choices and unexpected problems. First-time dog owners are more likely to experience certain canine behavioral problems, especially dominancerelated aggression, separation-related concerns, and overexcitability (Jagoe \& Serpell, 1996). It could be that individuals choose dogs that go on to exhibit those behaviors because they do not recognize them at the time of adoption, or they are unaware of the challenges such behaviors can pose in daily life.

\section{Limitations}

One of the largest challenges of working with existing data is the lack of control over the research setting. For example, the shelter's Match-Up II evaluations are used as a form of triage to identify behavior that might need correction and so affect what is done with the dog, which can change its behavior by the time of adoption from what was originally assessed. Dogs exhibiting severe responses will likely be recommended for an intensive intervention to correct negative responses, meaning that their behavioral tendencies would shift from intake to adoption, which could explain some of the mismatch we found between Match-Up II ratings and adopters' ratings.

Similarly, we were only able to assess adopters at one time point - the time of adoption. Many participants noted that they had "just met this animal" or were unsure in their choices - that is, they felt they had only observed some limited behavior rather than gained an understanding of the dog's overall personality. Given that this impression was sufficient to bring a new animal into one's home, it is still important to test how these initial impressions impact adoption choices. But future research should also examine whether the stated-revealed preference gap impacts satisfaction, by doing follow-up assessment with adopters - that is, even if individuals choose animals inconsistent with their stated preferences at the time of adoption, does that matter, in terms of adopter satisfaction, after a period of time together at home has passed? Furthermore, follow-ups could evaluate whether individuals actually do succeed in choosing a dog that, in terms of its behavior in the home, meets their initial stated preferences.

Another challenge of field research is selection bias limiting the total range of behaviors seen. The dogs included in this work were those that were eventually adopted, and not every dog in the chosen field site shared that outcome. Although this shelter did not practice space-saving euthanasia on dogs during the study period, animals with behavior that would endanger the public might have been removed from the population prior to a Match-Up II assessment, eliminating some of the extreme variation we would expect on a behavior evaluation, especially in terms of aggression. Match-Up II also limits the amount of aggressive behaviors exhibited by ending a subtest once one of five behaviors deemed aggressive are shown, meaning that the range of aggression on the test is highly limited. We further found a substantial floor effect in many behaviors, where most dogs did not exhibit the majority of behaviors available, which also limits predictive power.

\section{Conclusion}

Adopting dogs is an important and relatively common real-world decision that seems ripe for enrichment using big data. In our study, adopters exhibited relatively small stated-revealed preference gaps, but it remains unclear whether this conclusion is wellsupported. Many of the data available within shelters may only offer limited insight into what behaviors impact the choice to adopt an animal, suggesting a more complicated pattern than can be captured through big or existing data alone, and pointing to the need to combine such data with purposely collected measures.

Author note The first author is a volunteer at the field site in this study, but received no financial compensation. We thank our field site for granting access to their facility and the data utilized in this work. This work was supported in part by an Indiana University Graduate and Professional Student Government Research Award granted to the first author. None of the data or materials for the experiments reported here are available, and none of the experiments were preregistered.

\section{References}

American Humane Association. (2013). Keeping pets (dogs and cats) in homes: A three-phase retention study. Phase II: Descriptive study of post-adoption retention in six shelters in three U.S. cities. Washington, DC: American Humane Association. Retrieved from https://www.americanhumane.org/app/uploads/2016/08/petsmartkeeping-pets-phase-ii.pdf

American Veterinary Medical Association. (2012). US pet ownership and demographics sourcebook. Schaumburg, IL: American Veterinary Medical Association.

Animal Rescue League of Boston@ (2016). Match-Up II® Shelter Dog Rehoming Program. Boston, MA: Animal Rescue League of Boston@. https://www.arlboston.org/wp-content/uploads/2017/01/ ReconstuctionManual 2016.pdf. Accessed 14 Dec 2016

Barker, S. B., \& Barker, R. T. (1988). The human-canine bond: Closer than family ties? Journal of Mental Health Counseling, 10, 46-56.

Beshears, J., Choi, J. J., Laibson, D., \& Madrian, B. C. (2008). How are preferences revealed? Journal of Public Economics, 92, 1787-1794.

Bramlett, M. D., \& Mosher, W. D. (2002). Cohabitation, marriage, divorce, and remarriage in the United States. Vital Health Statistics, $23,1-32$.

Buss, D. (1989). Sex differences in human mate preferences: Evolutionary hypotheses tested in 37 cultures. Behavioral and Brain Sciences, 12,1-14. https://doi.org/10.1017/ S0140525X00023992 
Cavanaugh, L. A., Leonard, H. A., \& Scammon, D. L. (2008). A tail of two personalities: How canine companions shape relationships and well-being. Journal of Business Research, 61, 469-479.

DeLeeuw, J. L. (2010). Animal shelter dogs: Factors predicting adoption versus euthanasia (Doctoral dissertation). Wichita State University, Wichita, KS.

Eastwick, P. W., \& Finkel, E. J. (2008). Sex differences in mate preferences revisited: Do people know what they initially desire in a romantic partner? Journal of Personality and Social Psychology, 94, 245-264. https://doi.org/10.1037/0022-3514.94.2.245

Finkel, E. J., Eastwick, P. W. \& Matthews, J. (2007). Speed-dating as an invaluable tool for studying romantic attraction: A methodological primer. Personal Relationships, 14, 149-166. https://doi.org/10. 1111/j.1475-6811.2006.00146.x

Furman, W. (2002). The emerging field of adolescent romantic relationships. Current Directions in Psychological Science, 11, 177-180.

Jagoe, A., \& Serpell, J. (1996). Owner characteristics and interactions and the prevalence of canine behaviour problems. Applied Animal Behaviour Science, 47, 31-42.

Jones, A. C. (2008). Development and validation of a dog personality questionnaire (Doctoral dissertation). University of Texas at Austin, TX.

Kurzban, R., \& Weeden, J. (2005). HurryDate: Mate preferences in action. Evolution and Human Behavior, 26, 227-244. https://doi.org/ 10.1016/j.evolhumbehav.2004.08.012

Lepper, M., Kass, P. H., \& Hart, L. A. (2002). Prediction of adoption versus euthanasia among dogs and cats in a California animal shelter. Journal of Applied Animal Welfare Science, 5, 29-42.

Levine, D. W. (2005). Do dogs resemble their owners? A reanalysis of Roy and Christenfeld (2004). Psychological Science, 16, 83-84. https://doi.org/10.1111/j.0956-7976.2005.00785.x

Li, N. P., Bailey, J. M., Kenrick, D. T., \& Linsenmeier, J. A. W. (2002). The necessities and luxuries of mate preferences: Testing the tradeoffs. Journal of Personality and Social Psychology, 82, 947955. https://doi.org/10.1037/0022-3514.82.6.947

Li, N. P., Yong, J. C., Tov, W., Sng, O., Fletcher, G. J. O., Valentine, K. A., ... Balliet, D. (2013). Mate preferences do predict attraction and choices in the early stages of mate selection. Journal of Personality and Social Psychology, 105, 757-776. https://doi.org/ 10.1037/a0033777

Lykken, D. T., \& Tellegen, A. (1993). Is human mating adventitious or the result of lawful choice? A twin study of mate selection. Journal of Personality and Social Psychology, 65, 56-68. https://doi.org/10. 1037/0022-3514.65.1.56

Marston, L. C., Bennett, P. C., \& Coleman, G. J. (2005). Adopting shelter dogs: Owner experiences of the first month post-adoption. Anthrozoös, 18, 358-378.

Mirko, E., Doka, A., \& Miklosi, A. (2013). Association between subjective rating and behavior coding and the role of experience in making video assessments on the personality of the domestic dog (Canis familiaris). Applied Animal Behaviour Science, 149, 45-54

Mohan-Gibbons, H., Dolan, E. D., Reid, P., Slater, M. R., Mulligan, H., \& Weiss, E. (2018). The impact of excluding food guarding from a standardized behavioral canine assessment in animal shelters. Animals, 8(2). https://doi.org/10.3390/ani8020027

Nakajima, S., Yamamoto, M., \& Yoshimoto, N. (2009). Dogs look like their owners: Replications with racially homogeneous owner portraits. Anthrozoös, 22, 173-181.

Nisbett, R. E., \& Wilson, T. D. (1977). Telling more than we can know: Verbal reports on mental processes. Psychological Review, 84, 231 259. https://doi.org/10.1037/0033-295X.84.3.231

Patronek, G. J., \& Bradley, J. (2016). No better than flipping a coin: Reconsidering canine behavior evaluations in animal shelters.
Journal of Veterinary Behavior: Clinical Applications and Research, 15, 66-77.

Penke, L., Todd, P. M., Lenton, A. P., \& Fasolo, B. (2007). How selfassessments can guide human mating decisions. In G. Geher \& G. Miller (Eds.), Mating intelligence: Sex, relationships, and the mind's reproductive system (pp. 37-75). New York, NY: Erlbaum.

Posage, J. M., Bartlett, P. C., \& Thomas, D. K. (1998). Determining factors for successful adoption of dogs from an animal shelter. Journal of the American Veterinary Medical Association, 213, 478-482.

Riefer, P. S., Prior, R., Blair, N., Pavey, G., \& Love, B. C. (2017). Coherencymaximizing exploration in the supermarket. Nature Human Behaviour, 1, 17. https://doi.org/10.1038/s41562-016-0017

Roy, M. M., \& Christenfeld, N. J. (2004). Do dogs resemble their owners? Psychological Science, 15, 361-363.

Roy, M. M., \& Christenfeld, N. J. (2005). Dogs still do resemble their owners. Psychological Science, 16, 743-744.

Seabold, S., \& Perktold, J. (2010). Statsmodels: Econometric and statistical modeling with Python. In S. van der Walt \& J. Millman (Eds.), Proceedings of the 9th Python in Science Conference (pp. 57-61). Retrieved from http://conference.scipy.org/proceedings/scipy2010/ seabold.html

Shackelford, T. K., Schmitt, D. P., \& Buss, D. M. (2005). Universal dimensions of human mate preferences. Personality and Individual Differences, 39, 447-458.

Shelter Animals Count. (2016). 2016 animal sheltering statistics. Montclair, NJ: Author. Retrieved May 6, 2018, from https:// shelteranimalscount.org/docs/default-source/DataResources/ 2016animalshelteringstatistics.pdf?sfvrsn $=12$

Siettou, C., Fraser, I. M., \& Fraser, R. W. (2014). Investigating some of the factors that influence "consumer" choice when adopting a shelter dog in the United Kingdom. Journal of Applied Animal Welfare Science, 17, 136-147.

Tami, G., \& Gallagher, A. (2009). Description of the behaviour of domestic dog (Canis familiaris) by experienced and inexperienced people. Applied Animal Behaviour Science, 120, 159-169.

Thiessen, D., \& Gregg, B. (1980). Human assortative mating and genetic equilibrium: An evolutionary perspective. Ethology and Sociobiology, 1, 111-140.

Todd, P. M., Penke, L., Fasolo, B., \& Lenton, A. P. (2007). Different cognitive processes underlie human mate choices and mate preferences. Proceedings of the National Academy of Sciences, 104, 15011-15016.

Turcsán, B., Range, F., Virányi, Z., Miklósi, Á., \& Kubinyi, E. (2012). Birds of a feather flock together? Perceived personality matching in ownerdog dyads. Applied Animal Behaviour Science, 140, 154-160.

Watson, D., Klohnen, E. C., Casillas, A., Nus Simms, E., Haig, J., \& Berry, D. S. (2004). Match makers and deal breakers: Analyses of assortative mating in newlywed couples. Journal of Personality, 72, 1029-1068.

Weiss, E., Miller, K., Mohan-Gibbons, H., \& Vela, C. (2012). Why did you choose this pet? Adopters and pet selection preferences in five animal shelters in the United States. Animals, 2, 144-159.

Wells, D., \& Hepper, P. G. (1992). The behaviour of dogs in a rescue shelter. Animal Welfare, 1, 171-186.

Publisher's note Springer Nature remains neutral with regard to jurisdictional claims in published maps and institutional affiliations. 